\title{
Next Generation Sequencing methods for identification of mutations and large structural variants, 11 - 12 March 2014
}

Vital-IT and the Bioinformatics Unravelling groups of the SIB Swiss Institute of Bioinformatics are organising a joint ALLBIO and COST SeqAhead event in Lausanne, Switzerland

Grégoire Rossier (AllBio partner) \& Laurent Falquet (SeqAhead partner) are pleased to announce a joint ALLBIO and COST SeqAhead event in Lausanne, Switzerland.

It is entitled "Next Generation Sequencing (NGS) methods for identification of mutations and large structural variants" and will be held from Tuesday 11 March to Wednesday 12 March, 2014.

This international event covers several aspects of the identification of genomic structural variants using NGS data. The mini symposium (Day 1) will present the latest developments in the field and the workshop (Day 2) will allow participants to get used to the tools with a virtual machine prepared during a test case hackathon. Particular emphasis will be given to the comparison of the different analysis tools and how to combine their results.

The objective of the mini symposium is to provide an overview of the existing tools/pipelines available for NGS analysis, as well as to present some data using those tools. The objective of the workshop is to allow participants using the pipeline, either with our data, or with their own data.

\section{Requirements for the workshop:}

- Basic knowledge of UNIX

- A laptop with at least 4 GB RAM, 50 GB of free disk space, WIFI and VirtualBox preinstalled

The number of seats is limited to 90 for the mini symposium and to 25 for the workshop. Further information and application are available from http://edu.isb-sib.ch/course/view.php?id=104.

ALLBIO (http://www.allbioinformatics.eu) is the main sponsor of this event, and in addition 10 seats of the workshop are sponsored by the COST Action SeqAhead (http://www.segahead.eu).

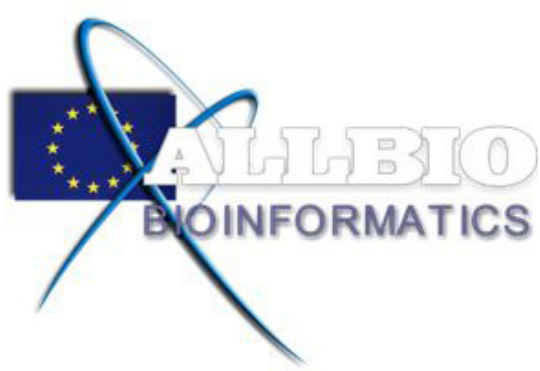

\section{SeqAhead}

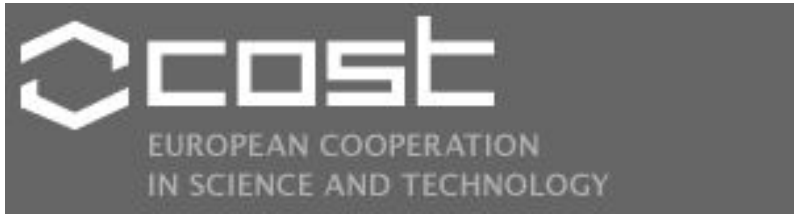

